\title{
Manufacturers push the limits of megacombo vaccines for kids
}

If the pain inflicted by the annual flu shot seems bad, pity what babies endure: in the US, childhood immunization guidelines call for up to 19 separate injections in the first six months of an infant's life. And although the vast majority of parents follow the recommendations, nearly half still report concerns about the associated pain for their children, according to a recent survey conducted by the US Centers for Disease Control and Prevention.

To bring down the number of injectionsand subsequently hopefully boost compliance rates even further-drugmakers have developed several combination vaccines that provide protection against multiple diseases in a single shot. For the past decade, the EU, Canada and elsewhere have had a vaccine called Infanrix hexa, manufactured by GlaxoSmithKline (GSK), that protects against six diseases. And in late February, the European Medicines Agency's Committee for Medicinal Products for Human Use reached a decision in support of licensure in the majority of European countries for a similar product from Sanofi Pasteur.

Both six-in-one vaccines include antigens designed to prevent diphtheria, tetanus, pertussis, hepatitis $\mathrm{B}$, polio and serious illness caused by the bacterium Haemophilus influenzae type b. These six-in-one vaccines have not been submitted for approval in the US, but the country has two vaccines that each protect against five diseases.

With clear advantages provided by these combination products, and little evidence of added safety risks, the question now being asked by some pediatricians and vaccine manufacturers is: how many diseases can you protect against in a single jab while still eliciting proper immune responses and maintaining adequate safety profiles? "The experience of developing five-fold or six-fold combinations has been that they're not easy to make," says Stanley Plotkin, former medical and scientific director of Pasteur-Mérieux-Connaught (now Sanofi Pasteur, based in Lyon, France), who runs a vaccine consultancy in Doylestown, Pennsylvania. "But from a theoretical point of view, there is not yet a limitation on the number of vaccines you could put together in the same syringe."

Despite the fact that some parents, scared by now-debunked ties between the measles, mumps, and rubella 'MMR' combination vaccine and autism, have sought separate vaccines in the past, researchers assert that the combinations are safe. "From an immunological point of view, there's no harm to increasing the valency to the multitude that we have today

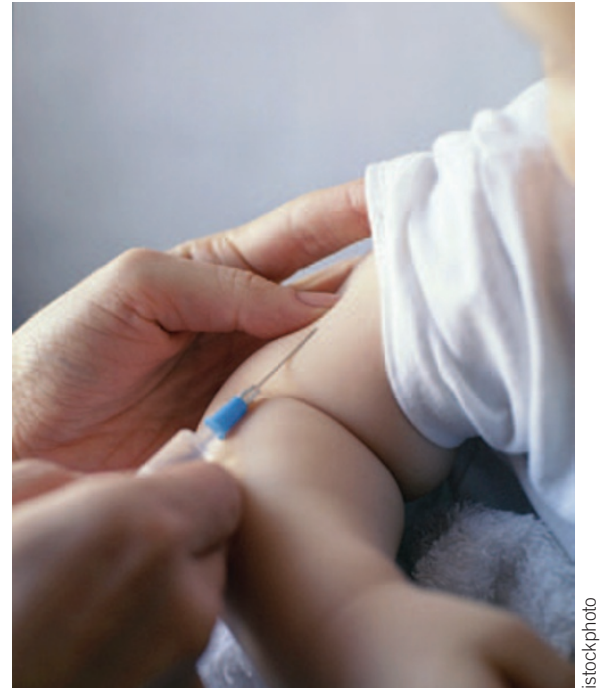

This will hurt: But less often.

because there's quite a bit of evidence that the immune system can handle a large number of antigens at any one time," says Hugues Bogaerts, former senior medical director at GSK Biologicals (a division of the UK-based drug giant) who helped develop Infanrix and now works as a consultant near Brussels.

\section{A busy calendar}

For drug companies, the value added in megavaccines means that they generally command slightly higher prices than if each shot was sold individually. And for parents and public health agencies, a vaccine that contains antigens against multiple diseases could help simplify an increasingly complicated immunization schedule.

"The benefit is definitely compliance," says Courtney Gidengil, a pediatric infectious disease physician at Boston Children's Hospital who studies issues surrounding vaccine implementation at the RAND Corporation, a policy think tank headquartered in Santa Monica, California. "If someone balks at three shots, you can give this one instead and they will be hopefully more willing to accept it. Plus, they don't have to come back another time, forget and then fall behind."

In the future, the immunization schedule will inevitably become even more unwieldy as new pediatric vaccines prove successful against further infectious diseases. For example, the first meningitis B vaccine-Bexsero, from Switzerland's Novartis-won approval in Europe earlier this year and will soon join the roster of early childhood vaccines in many EU countries. "If there is again a shortage of space in the vaccination calendar, this could eventually encourage researchers, manufacturers, regulatory authorities and public health officers that we may have to go beyond six [diseases in one shot] for the sake of optimizing our calendar," Bogaerts says.

But combining even more antigens to make a seven- or eight-in-one megavaccine presents an even tougher technical challenge. "The more diseases you're working with, the more complex the formulations," says Christian Steber, a project manager at Sanofi Pasteur's US headquarters in Swiftwater, Pennsylvania, who is working on a new six-arm vaccine known interchangeably as PR5I or V419 that is currently in phase 3 trials in the US and the EU. Every step to introduce an additional type of antigen "may result in an additional number of quality control steps, and quality control today takes longer than actual production," Bogaerts adds. "Just mathematically, the number of steps increases, and then the risk that something goes wrong is not increasingly linearly, but exponentially."

Then there are logistical hurdles that sit outside the control of a vaccine maker's hands. "It's a very complex equation that includes inputs from a public health perspective, and one has to think about aligning vaccination schedules, which are not necessarily the same in different geographic regions," says Jim Tartaglia, project head for new vaccines for Sanofi Pasteur's North American operations.

That's what ultimately sank GSK2202083A, the only seven-in-one vaccine clinically tested to date, according to its developers. The vaccine, from GSK Biologicals, targeted all the same diseases as Infanrix hexa in addition to meningitis $\mathrm{C}$, and it went through multiple phase 2 trials in Canada and Europe, the results of which are expected to be published in July. However, according to Melinda Stubbee, a GSK spokesperson, the company did not advance the product further because many countries reduced the recommended number of meningitis $\mathrm{C}$ shots, which meant that the timing and frequency of meningitis vaccinations did not coincide with the other components of the product. "We continue to review the potential of combination vaccines as part of our development plan as a means to improve compliance to vaccination and reduce the number of injections," Stubbee says.

According to Tartaglia, Sanofi Pasteur does not currently have a vaccine with protection against more than six diseases in the works either. To do so, he says, "the stars have to align around all of the aspects in moving forward."

Elie Dolgin 